Research Article

\title{
Complete Convergence for Weighted Sums of Widely Acceptable Random Variables under Sublinear Expectations
}

\author{
Rong Hu and Qunying Wu \\ College of Science, Guilin University of Technology, Guilin 541004, China \\ Correspondence should be addressed to Qunying Wu; wqy666@glut.edu.cn
}

Received 2 February 2021; Revised 10 April 2021; Accepted 26 April 2021; Published 18 May 2021

Academic Editor: Fabio Tramontana

Copyright ( 2021 Rong Hu and Qunying Wu. This is an open access article distributed under the Creative Commons Attribution License, which permits unrestricted use, distribution, and reproduction in any medium, provided the original work is properly cited.

Using different methods than the probability space, under the condition that the Choquet integral exists, we study the complete convergence theorem for weighted sums of widely acceptable random variables under sublinear expectation space. We proved corresponding theorem which was extended to the sublinear expectations' space from the probability space, and similar results were obtained.

\section{Introduction}

In the study of probability theory and mathematical statistics, limit theory is an important research topic, which is widely used in the financial sector and other fields. However, the establishment of the classical limit theory requires strict conditions for the certainty model, especially in the practice of financial statistics and financial risk measurement, so its limitations are gradually highlighted by many uncertainties. Therefore, Peng [1-3] made an improvement, proposed a sublinear expectations' space that can model the probability and distribution of uncertainty, and gave the corresponding theoretical system, which has aroused the attention of the majority of scholars. At present, the limit theory has obtained many excellent results under sublinear expectation. For example, in the early research on sublinear expectation, Peng [1-3] extended the central limit theory in the traditional probability space to the sublinear expectation space. Zhang [4-6] continued his research on extended negatively dependent random variables and obtained Kolmogorov's strong law of large numbers (SLLN) and a series of inequalities under sublinear expectation. Zhang and Chen [7] obtain the central limit theorem for weighted sums in sublinear expectations' space. Feng et al. [8] obtain a complete convergence of the weighted sum of negatively dependent (ND) sequences in the sublinear expectations' space. Wang and $\mathrm{Wu}$ [9] study on complete convergence and almost sure convergence under the sublinear expectations. Chen [10] obtains a SLLN for an independent identically distributed sequence in the sublinear expectations space. Liang and $\mathrm{Wu}[11]$ research on complete convergence and complete integral convergence for extended negatively dependent (END) random variables under sublinear expectations.

Complete convergence is one of the most important problems in limit theory research because of the extensive application of weighted sum in statistics, and its properties attract more scholars to study and discuss. In the study of complete convergence in probability space, statistician Hsu and Robbins [12] first propose the concept of complete convergence in 1947, which aroused the interest of many scholars. So far, complete convergence has been studied very deeply in probability space, for example, Liang and Su [13] obtain the complete convergence theorem for weighted sums of negatively associated (NA) sequences and discuss its necessity. Sung [14], based on the exponential inequality, obtains new complete convergence results for weighted sums of independent random variables. $\mathrm{Wu}[15,16]$ proves the complete convergence theorems for ND sequences and arrays of row-wise ND random variables. Lita [17] explores 
the property of complete convergence for END random variables. In this article, we establish the complete convergence theorem for weighted sums of widely acceptable (WA) random variables under sublinear expectations. The results have been obtained by Lang et al. [18] and have been generalized to the sublinear expectation space.

\section{Preliminaries}

We use the framework and notations of Peng [1-3]. Let $(\Omega, \mathscr{F})$ be a given measurable space and let $\mathscr{H}$ be a linear space of real functions defined on $(\Omega, \mathscr{F})$ such that if $X_{1}, X_{2}, \ldots, X_{n} \in \mathscr{H}$, then $\varphi\left(X_{1}, \ldots, X_{n}\right) \in \mathscr{H}$ for each $\varphi \in C_{l \text { Lip }}\left(\mathbb{R}_{n}\right)$, where $C_{l \text {,Lip }}\left(\mathbb{R}_{n}\right)$ denotes the linear space of (local Lipschitz) functions $\varphi$ satisfying

$$
|\varphi(\mathbf{x})-\varphi(\mathbf{y})| \leq c\left(1+|\mathbf{x}|^{m}+|\mathbf{y}|^{m}\right)|\mathbf{x}-\mathbf{y}|, \quad \forall \mathbf{x}, \mathbf{y} \in \mathbb{R}_{n},
$$

for some $c>0, m \in \mathbb{N}$ depending on $\varphi . \mathscr{H}$ is considered as a space of random variables. In this case, we denote $X \in \mathscr{H}$.

Definition 1 (see [2]). A sublinear expectation $\widehat{E}$ on $\mathscr{H}$ is a function $\widehat{E}: \mathscr{H} \longrightarrow \overline{\mathbb{R}}$ satisfying the following properties: for all $X, Y \in \mathscr{H}$, we have

(a) Monotonicity: if $X \geq Y$, then $\widehat{E} X \geq \widehat{E} Y$

(b) Constant preserving: $\widehat{E} c=c$

(c) Subadditivity: $\widehat{E}(X+Y) \leq \widehat{E} X+\widehat{E} Y$, whenever $\widehat{E} X+$ $\widehat{E} Y$ is not of the form $+\infty-\infty$ or $-\infty+\infty$

(d) Positive homogeneity: $\widehat{E}(\lambda X)=\lambda \widehat{E} X, \lambda \geq 0$

Here, $\overline{\mathbb{R}}=[-\infty, \infty]$. The triple $(\Omega, \mathscr{H}, \widehat{E})$ is called a sublinear expectation space.

Given a sublinear expectation $\widehat{E}$, let us denote the conjugate expectation $\widehat{\mathcal{E}}$ of $\widehat{E}$ by

$$
\widehat{\varepsilon} X:=-\widehat{E}(-X), \quad \forall X \in \mathscr{H} .
$$

From the definition, it is easily shown that, for all $X, Y \in \mathscr{H}$,

$$
\begin{gathered}
\hat{\mathcal{E}} X \leq \hat{E} X, \\
\widehat{E}(X+c)=\widehat{E} X+c, \\
|\widehat{E}(X-Y)| \leq \widehat{E}|X-Y|, \\
\widehat{E}(X-Y) \geq \widehat{E} X-\widehat{E} Y .
\end{gathered}
$$

If $\widehat{E} Y=\widehat{\varepsilon} Y$, then $\widehat{E}(X+a Y)=\widehat{E} X+a \widehat{E} Y$ for any $a \in \mathbb{R}$. Next, we consider the capacities corresponding to the sublinear expectations. Let $\mathscr{G} \subset \mathscr{F}$. A function $V: \mathscr{G} \longrightarrow[0,1]$ is called a capacity if

$$
\begin{aligned}
& V(\varnothing)=0, \\
& V(\Omega)=1, \\
& V(A) \leq V(B), \quad \text { for } \forall A \subseteq B, A, B \in \mathscr{G} .
\end{aligned}
$$

It is called subadditive if $V(A \cup B) \leq V(A)+V(B)$ for all $A, B \in \mathscr{G}$ with $A \cup B \in \mathscr{G}$. In the sublinear space $(\Omega, \mathscr{H}, \widehat{E})$, we denote a pair $(\mathbb{V}, \mathscr{V})$ of capacities by

$$
\begin{aligned}
& \mathbb{V}(A):=\inf \{\hat{\mathbb{E}} \xi ; I(A) \leq \xi, \xi \in \mathscr{H}\}, \\
& \mathscr{V}(A):=1-\mathbb{V}\left(A^{c}\right), \quad \forall A \in \mathscr{F},
\end{aligned}
$$

where $A^{c}$ is the complement set of $A$. By definition of $\mathbb{V}$ and $\mathscr{V}$, it is obvious that $\mathbb{V}$ is subadditive, and

$$
\mathscr{V}(A) \leq \mathbb{V}(A), \quad \forall A \in \mathscr{F} .
$$

If $I(A) \in \mathscr{H}$, then

$$
\begin{aligned}
\mathbb{V}(A) & =\widehat{E}(I(A)), \\
\mathscr{V}(A) & =\widehat{\mathcal{\varepsilon}}(I(A)) .
\end{aligned}
$$

If $f \leq I(A) \leq g, f, g \in \mathscr{H}$, then

$$
\widehat{E} f \leq \mathbb{V}(A) \leq \widehat{E} g, \quad \widehat{\varepsilon} f \leq \mathscr{V}(A) \leq \widehat{\varepsilon} g .
$$

This implies Markov inequality: $\forall X \in \mathscr{H}$,

$$
\mathbb{V}(|X| \geq x) \leq \frac{\widehat{E}\left(|X|^{p}\right)}{x^{p}}, \quad \forall x>0, p>0,
$$

from $I(|X| \geq x) \leq\left(|X|^{p} / x^{p}\right) \in \mathscr{H}$.

Definition 2 (see [2]). We define the Choquet integrals/ expectations $\left(C_{\mathbb{V}}, C_{\mathscr{V}}\right)$ by

$$
C_{\mathbb{V}}(X)=\int_{0}^{\infty} V(X \geq t) \mathrm{d} t+\int_{-\infty}^{0}[V(X \geq t)-1] \mathrm{d} t,
$$

with $V$ being replaced by $\mathbb{V}$ and $\mathscr{V}$, respectively.

Definition 3 (see [2]).

(i) $\widehat{E}$ countably subadditive: $\widehat{E}$ is called to be countably subadditive if it satisfies

$$
\widehat{E}(X) \leq \sum_{n=1}^{\infty} \widehat{E}\left(X_{n}\right), \quad \text { where } X \leq \sum_{n=1}^{\infty} X_{n}, X, X_{n} \in \mathscr{H}, X \geq 0, X_{n} \geq 0, n \geq 1
$$

(ii) $V$ is called to be countably subadditive if

$$
V\left\{\bigcup_{n=1}^{\infty} A_{n}\right\} \leq \sum_{n=1}^{\infty} V\left(A_{n}\right), \quad \forall A_{n} \in \mathscr{F}
$$

Definition 4 (identical distribution, see [2]). Let $\mathbf{X}_{1}$ and $\mathbf{X}_{2}$ be two $n$-dimensional random vectors defined, respectively, in a sublinear expectation spaces $\left(\Omega_{1}, \mathscr{H}_{1}, \widehat{E}_{1}\right)$ and $\left(\Omega_{2}, \mathscr{H}_{2}, \widehat{E}_{2}\right)$. They are called identically distributed if 


$$
\widehat{E}\left[\varphi\left(\mathbf{X}_{1}\right)\right]=\widehat{E}\left[\varphi\left(\mathbf{X}_{2}\right)\right], \quad \forall \varphi \in C_{l, \text { Lip }}\left(\mathbb{R}_{n}\right),
$$

whenever the sublinear expectation is finite. A sequence $\left\{X_{n} ; n \geq 1\right\}$ of random variables is said to be identically distributed if, for each $i \geq 1, X_{i}$ and $X_{1}$ are identically distributed.

Definition 5 (widely acceptable, see [19]). Let $\left\{Y_{n}, n \geq 1\right\}$ be a sequence of random variables in a sublinear expectation $\operatorname{space}(\Omega, \mathscr{H}, \hat{\mathbb{E}})$. The sequence $\left\{Y_{n}, n \geq 1\right\}$ is called widely acceptable (WA) if for $t \geq 0$, and for all $n \in \mathbb{N}$,

$$
\hat{\mathbb{E}} \exp \left(\sum_{i=1}^{n} t Y_{i}\right) \leq g(n) \prod_{i=1}^{n} \hat{\mathbb{E}} \exp \left(t Y_{i}\right)
$$

where $0<g(n)<\infty$.

It is obvious that if $\left\{X_{n} ; n \geq 1\right\}$ is a sequence of widely acceptable random variables and functions $f_{1}(x), f_{2}(x), \ldots, \in C_{l, \text { Lip }}\left(\mathbb{R}_{n}\right)$ are all nondecreasing (resp. all nonincreasing), then $\left\{f_{n}\left(X_{n}\right) ; n \geq 1\right\}$ is also a sequence of widely acceptable random variables.

In the following, let $\left\{X_{n} ; n \geq 1\right\}$ be a sequence of random variables in $(\Omega, \mathscr{H}, \hat{\mathbb{E}})$, and $S_{n}=\sum_{i=1}^{n} X_{i}$. The symbol $c$ stands for a generic positive constant which may differ from one place to another. Let $a_{x} \sim b_{x}$ denote $\lim _{x \rightarrow \infty} a_{x} / b_{x}=1$. $a_{x} \ll b_{x}$ denotes that there exists a constant $c>0$ such that $a_{x} \leq c b_{x}$ for sufficiently large $x$, and $I(\cdot)$ denotes an indicator function.

To prove our results, we need the following two lemmas.

Lemma 1 (see [19]). Let $\left\{X_{1}, \ldots, X_{n}\right\}$ be a sequence of $W A$ random variables in $(\Omega, \mathscr{H}, \hat{\mathbb{E}})$, with $\hat{\mathbb{E}}\left(X_{k}\right) \leq 0$ for $1<i \leq n$. Then, for all $x>0, d>0$, we have

$$
\mathbb{V}\left(S_{n} \geq x\right) \leq \mathbb{V}\left(\max _{1 \leq i \leq n} X_{i}>d\right)+g(n) \exp \left(\frac{x}{d}-\frac{x}{d} \ln \left(1+\frac{x d}{\sum_{i=1}^{n} \hat{\mathbb{E}}\left|X_{i}\right|^{2}}\right)\right)
$$

Proof. We can easily get (15) from Theorem 1 of [19].

Lemma 2 Suppose $X \in H, p>0$, for any constant $c>0$ :

(i) Then,

$$
C_{\mathbb{V}}\left(|X|^{p}\right)<\infty \Leftrightarrow \sum_{n=1}^{\infty} n^{p-1} \mathbb{V}(|X|>c n)<\infty .
$$

Especially, when $p=1$, we have

$$
C_{\mathbb{V}}(|X|)<\infty \Leftrightarrow \sum_{n=1}^{\infty} \mathbb{V}(|X|>c n)<\infty .
$$

(ii) If $C_{\mathbb{V}}\left(|X|^{p}\right)<\infty$, then, for any $a>1$,

$$
\sum_{k=1}^{\infty} a^{k} \mathbb{V}\left(|X|>c a^{(k / p)}\right)<\infty .
$$

Proof. For (i), similar to the proof of Lemma 3 in [9] and assuming $x^{(1 / p)}=y$, then (16) is obtained. For (ii), similar to the proof Theorem 6 in [20], we can get, for any $a>1,(18)$ is satisfied.

\section{The Main Results and Their Proofs}

(A.1) Let $g(x)$ be a nondecreasing positive function on $[0, \infty), g(x)=g(n)$, when $x=n$. And, $g(x) / x^{\tau} \downarrow$ for some $0<\tau<1$

(A.2) There exists a nondecreasing positive function $h(x)$ on $[0, \infty)$, such that $h(x) / x \downarrow$ and $\sum_{n=1}^{\infty} g(n) /\left(n h^{\gamma}(\mu n)\right)<\infty$ for some $\gamma>0,0<\mu<1$
Theorem 1. Let $\left\{X_{n} ; n \geq 1\right\}$ be a sequence of $W A$ and identically distributed random variables under sublinear expectations. $g(x)$ satisfies (A.1) or (A.2), and $p \geq 1$. When $p=1$, assume that (A.1) holds and

$$
\hat{\mathbb{E}}\left(|X|^{1+\delta}\right) \leq C_{\mathbb{V}}\left(|X|^{1+\delta}\right)<\infty,
$$

for some $\delta>0$, or (A.2) holds and

$$
\hat{\mathbb{E}}(|X| h(|X|)) \leq C_{\mathbb{V}}(|X| h(|X|))<\infty .
$$

When $p>1$, assume that

$$
\hat{\mathbb{E}}\left(|X|^{p}\right) \leq C_{\mathbb{V}}\left(|X|^{p}\right)<\infty,
$$

and (A.1) or (A.2) holds. Let $\left\{a_{n i} ; 1 \leq i \leq n, n \geq 1\right\}$ be an array of real positive numbers satisfying

$$
\max _{1 \leq i \leq n} a_{n i}=O\left(n^{-1}\right) \text {. }
$$

Then, for all $\varepsilon>0$,

$$
\begin{gathered}
\sum_{n=1}^{\infty} n^{p-2} \mathbb{V}\left(\sum_{i=1}^{n} a_{n i}\left(X_{i}-\hat{\mathbb{E}} X_{i}\right)>\varepsilon\right)<\infty, \\
\sum_{n=1}^{\infty} n^{p-2} \mathbb{V}\left(\sum_{i=1}^{n} a_{n i}\left(X_{i}-\hat{\varepsilon} X_{i}\right)<-\varepsilon\right)<\infty .
\end{gathered}
$$

In particular, if $\hat{\mathbb{E}} X_{i}=\widehat{\varepsilon} X_{i}$, then

$$
\sum_{n=1}^{\infty} n^{p-2} \mathbb{V}\left(\left|\sum_{i=1}^{n} a_{n i}\left(X_{i}-\hat{\varepsilon} X_{i}\right)\right|>\varepsilon\right)<\infty
$$


Proof. To simplify our proof, we may assume that $\max _{1 \leq i \leq n} a_{n i} \leq n^{-1}$; thus,

$$
\sum_{i=1}^{n} a_{n i}^{2} \leq\left(\max _{1 \leq i \leq n} a_{n i}\right)^{2} \sum_{i=1}^{n} 1 \leq n^{-1} .
$$

When $p=1$, it is obvious that $C_{\mathbb{V}}(|X|)<\infty$ by $C_{\mathbb{V}}\left(|X|^{1+\delta}\right)<\infty \quad$ or $\quad C_{\mathbb{V}}(|X| h(|X|))<\infty$. When $p>1$, $C_{\mathbb{V}}(|X|)<\infty$ follows from $C_{\mathbb{V}}\left(|X|^{p}\right)<\infty$. Therefore, there exists a positive integer $N$ such that

$$
\hat{\mathbb{E}}|X| I(|X|>N)<\frac{\varepsilon}{6} .
$$

For widely acceptable random variables $\left\{X_{n} ; n \geq 1\right\}$, in order to ensure that the truncated random variables are also widely acceptable, truncated functions should belong to $C_{l, \text { Lip }}$ and should be nondecreasing. Denote for $1 \leq i \leq n$ that

$$
\begin{aligned}
X_{i}^{\prime} & =N I\left(X_{i}>N\right)+X_{i} I\left(\left|X_{i}\right| \leq N\right)-N I\left(X_{i}<-N\right), \\
X_{i}^{\prime \prime}=X_{i}-X_{i}^{\prime} & =\left(X_{i}-N\right) I\left(X_{i}>N\right)+\left(X_{i}+N\right) I\left(X_{i}<-N\right) .
\end{aligned}
$$

It is easily checked that

$$
\begin{aligned}
& \sum_{n=1}^{\infty} n^{p-2} \mathbb{V}\left(\sum_{i=1}^{n} a_{n i}\left(X_{i}-\hat{\mathbb{E}} X_{i}\right)>\varepsilon\right) \\
= & \sum_{n=1}^{\infty} n^{p-2} \mathbb{V}\left(\left(\sum_{i=1}^{n} a_{n i}\left(X_{i}^{\prime}-\hat{\mathbb{E}} X_{i}^{\prime}\right)+\sum_{i=1}^{n} a_{n i} X_{i}^{\prime \prime}+\sum_{i=1}^{n} a_{n i}\left(\hat{\mathbb{E}} X_{i}^{\prime}-\hat{\mathbb{E}} X_{i}\right)\right)>\varepsilon\right) \\
\leq & \sum_{n=1}^{\infty} n^{p-2} \mathbb{V}\left(\sum_{i=1}^{n} a_{n i}\left(X_{i}^{\prime}-\hat{\mathbb{E}} X_{i}^{\prime}\right)>\frac{\varepsilon}{3}\right)+\sum_{n=1}^{\infty} n^{p-2} \mathbb{V}\left(\sum_{i=1}^{n} a_{n i} X_{i}^{\prime \prime}>\frac{\varepsilon}{3}\right) \\
& +\sum_{n=1}^{\infty} n^{p-2} \mathbb{V}\left(\sum_{i=1}^{n} a_{n i}\left(\hat{\mathbb{E}} X_{i}^{\prime}-\hat{\mathbb{E}} X_{i}\right)>\frac{\varepsilon}{3}\right) \\
= & I_{1}+I_{2}+I_{3} .
\end{aligned}
$$

Thus, to prove the desired result (23), we only need to show $I_{1}<\infty, I_{2}<\infty$, and $I_{3}<\infty$.

Let $\left\{T_{i}, 1 \leq i \leq n, n \geq 1\right\}$ be a sequence of any random variable, and we all have

$$
\mathbb{V}\left(\max _{1 \leq i \leq n} T_{i}>d\right) \leq \sum_{i=1}^{n} \mathbb{V}\left(\left|T_{i}\right|>d\right) \leq \sum_{i=1}^{n} \frac{1}{d^{q}} \hat{\mathbb{E}}\left|T_{i}\right|^{q} .
$$

For each $n \geq 1,\left\{X_{i}^{\prime}, 1 \leq i \leq n\right\}$ is a sequence of WA, $\left\{X_{i}^{\prime}-\hat{\mathbb{E}} X_{i}^{\prime}\right\}$ satisfies the conditions of Lemma 1. Let $x=$ $(\varepsilon / 3)$ and $d=(\varepsilon /(6(p+\gamma)))$ in Lemma 1 ; taking $q>p$ and (30), we obtain

$$
\begin{aligned}
I_{1} \leq & \sum_{n=1}^{\infty} n^{p-2}\left(\sum_{i=1}^{n} \frac{1}{d^{q}} \hat{\mathbb{E}}\left|a_{n i}\left(X_{i}^{\prime}-\hat{\mathbb{E}} X_{i}^{\prime}\right)\right|^{q}\right) \\
& +g(n) \exp \left(\frac{\varepsilon}{3 d}-\frac{\varepsilon}{3 d} \ln \left(1+\frac{\varepsilon d}{3 \sum_{i=1}^{n} \hat{\mathbb{E}}\left|a_{n i}\left(X_{i}^{\prime}-\hat{\mathbb{E}} X_{i}^{\prime}\right)\right|^{2}}\right)\right)=: I_{11}+I_{12} .
\end{aligned}
$$

For $I_{11}$, it follows from the definition of $\left\{X_{i}^{\prime}, i \geq 1\right\}$ that $\hat{\mathbb{E}}\left|X_{i}^{\prime}\right| \leq N$. By Cr inequality and (22), we obtain 


$$
\begin{aligned}
I_{11} & \ll \sum_{n=1}^{\infty} n^{p-2} \sum_{i=1}^{n} \frac{1}{d^{q}} a_{n i}^{q} \hat{\mathbb{E}}\left|X_{i}^{\prime}\right|^{q} \\
& \leq c \sum_{n=1}^{\infty} n^{p-2} \sum_{i=1}^{n} a_{n i}^{q} \leq c \sum_{n=1}^{\infty} n^{p-2}\left(\max _{1 \leq i \leq n} a_{n i}\right)^{q} \sum_{i=1}^{n} 1 \\
& \leq c \sum_{n=1}^{\infty} n^{p-q-1} \\
& <\infty .
\end{aligned}
$$

For $I_{12}$, we get, by (26), Cr inequality, $\tau<1<1+\gamma$, and (A.1) or (A.2) holds, such that

$$
\begin{aligned}
& I_{12} \leq c \sum_{n=1}^{\infty} n^{p-2} g(n)\left(\sum_{i=1}^{n} \hat{\mathbb{E}}\left|a_{n i}\left(X_{i}^{\prime}-\hat{\mathbb{E}} X_{i}^{\prime}\right)\right|^{2}\right)^{(\varepsilon / 3 d)} \\
& \leq c \sum_{n=1}^{\infty} n^{p-2} g(n)\left(\sum_{i=1}^{n} a_{n i}^{2} \hat{\mathbb{E}}\left(X_{i}^{\prime}\right)^{2}\right)^{(\varepsilon / 3 d)} \\
& \leq c \sum_{n=1}^{\infty} n^{p-2} g(n)\left(\sum_{i=1}^{n} a_{n i}^{2}\right)^{(\varepsilon / 3 d)} \\
& \leq\left\{\begin{array}{l}
c \sum_{n=1}^{\infty} n^{p-2+\tau}\left(\sum_{i=1}^{n} a_{n i}^{2}\right)^{(\varepsilon / 3 d)}, \quad \text { if (A.1)holds, } \\
c \sum_{n=1}^{\infty} n^{p-2-(\varepsilon / 3 d)} \frac{g(n) h^{\gamma}(n) n^{\gamma+1}}{n h^{\gamma}(n) n^{\gamma}}, \quad \text { if (A.2)holds, }
\end{array}\right. \\
& \leq \begin{cases}c \sum_{n=1}^{\infty} n^{p-2+\tau-(\varepsilon / 3 d)}, & \text { if (A.1)holds, } \\
c \sum_{n=1}^{\infty} n^{p-1+\gamma-(\varepsilon / 3 d)}, & \text { if (A.2)holds, }\end{cases} \\
& = \begin{cases}c \sum_{i=1}^{\infty} n^{p-2+\tau-(\varepsilon /(3(\varepsilon /(6(p+\gamma))))}, & \text { if (A.1)holds, } \\
c \sum_{i=1}^{\infty} n^{p-1+\gamma-(\varepsilon /(3(\varepsilon /(6(p+\gamma))))}, & \text { if (A.2)holds, }\end{cases} \\
& \leq \sum_{n=1}^{\infty} n^{-1-p-\gamma}<\infty .
\end{aligned}
$$

Thus, we have proved $I_{1}<\infty$.

Now, we deal with $I_{2}$. For $n>N$, denote for $1 \leq i \leq n$ that

$$
\begin{aligned}
Y_{n i}= & \left(X_{i}-N\right) I\left(N<X_{i} \leq n\right)+(n-N) I\left(X_{i}>n\right) \\
& +\left(X_{i}+N\right) I\left(-n<X_{i} \leq-N\right)+(-n+N) I\left(X_{i}<-n\right) .
\end{aligned}
$$

Then,

$$
\begin{aligned}
I_{2} & =\sum_{n=1}^{\infty} n^{p-2} \mathbb{V}\left(\sum_{i=1}^{n} a_{n i} X_{i}^{\prime \prime}>\frac{\varepsilon}{3}\right) \\
& \leq \sum_{n=1}^{\infty} n^{p-2} \sum_{i=1}^{n} \mathbb{V}\left(\left|X_{i}\right|>n\right)+\sum_{n=1}^{\infty} n^{p-2} \sum_{i=1}^{n} \mathbb{V}\left(\sum_{i=1}^{n} a_{n i} Y_{n i}>\frac{\varepsilon}{3}\right) \\
& =I_{21}+I_{22} .
\end{aligned}
$$

For the $\mu$ of condition (A.2), let $z(x) \in C_{l, \text { Lip }}(\mathbb{R})$, $0 \leq z(x) \leq 1$, for all $x$, and $z(x)=1$ if $|x| \leq \mu, z(x)=0$ if $|x|>1$, and $z(x)$ are nonincreasing for any $x>0$. Then,

$$
\begin{aligned}
I(|x| \leq \mu) & \leq z(|x|) \leq I(|x| \leq 1), I(|x|>1) \\
& \leq 1-z(|x|) \leq I(|x|>\mu) .
\end{aligned}
$$

For $I_{21}$, we can get by (36); then,

$$
\begin{aligned}
I_{21} & \leq \sum_{n=1}^{\infty} n^{p-2} \sum_{i=1}^{n} \hat{\mathbb{E}}\left(1-z\left(\frac{\left|X_{i}\right|}{n}\right)\right) \\
& =\sum_{n=1}^{\infty} n^{p-2} \sum_{i=1}^{n} \hat{\mathbb{E}}\left(1-z\left(\frac{|X|}{n}\right)\right) \leq \sum_{n=1}^{\infty} n^{p-1} \mathbb{V}(|X|>\mu n) .
\end{aligned}
$$

When $p=1$, we can get $I_{21} \leq \sum_{n=1}^{\infty} \mathbb{V}(|X|>\mu n)<\infty$ by (17). When $p>1$, we can get $I_{21}<\infty$ by (16). Next, we prove $I_{22}<\infty$. Obviously,

$$
\begin{aligned}
I_{22}= & \sum_{n=1}^{\infty} n^{p-2} \mathbb{V}\left(\sum_{i=1}^{n} a_{n i} Y_{n i}>\frac{\varepsilon}{3}\right) \\
\leq & \sum_{n=1}^{\infty} n^{p-2} \mathbb{V}\left(\sum_{i=1}^{n} a_{n i}\left(Y_{n i}-\hat{\mathbb{E}} Y_{n i}\right)>\frac{\varepsilon}{6}\right) \\
& +\sum_{n=1}^{\infty} n^{p-2} \mathbb{V}\left(\sum_{i=1}^{n} a_{n i} \hat{\mathbb{E}} Y_{n i}>\frac{\varepsilon}{6}\right) .
\end{aligned}
$$

And, it follows from (22) and (27); when $n>N$, we have

$$
\begin{aligned}
\sum_{i=1}^{n} a_{n i} \hat{\mathbb{E}} Y_{n i} & \leq \sum_{i=1}^{n} a_{n i} \hat{\mathbb{E}}\left|X_{i}\right| I\left(\left|X_{i}\right|>N\right) \\
& \leq \max _{1 \leq i \leq n} a_{n i} \sum_{i=1}^{n} \hat{\mathbb{E}}|X| I(|X|>N) \\
& \leq \hat{\mathbb{E}}|X| I(|X|>N)<\frac{\varepsilon}{6} .
\end{aligned}
$$

So, we obtain $\mathbb{V}\left(\sum_{i=1}^{n} a_{n i} \hat{\mathbb{E}} Y_{n i}>(\varepsilon / 6)\right)=0$, when $n>N$. It follows that $\sum_{n=1}^{\infty} n^{p-2} \mathbb{V}\left(\sum_{i=1}^{n} a_{n i} \hat{\mathbb{E}} Y_{n i}>(\varepsilon / 6)\right)<\infty$. Thus, to estimate $I_{22}$, it suffices to show $\sum_{n=1}^{\infty} n^{p-2} \mathbb{V}\left(\sum_{i=1}^{n} a_{n i}\left(Y_{n i}-\hat{\mathbb{E}} Y_{n i}\right)>(\varepsilon / 6)\right)<\infty$. Next, we consider the following two cases. 
Case 1. $p>1$.

$\left\{Y_{n i}, i \geq 1, n>N\right\}$ also is a sequence of WA random variables, and $\left\{Y_{n i}-\hat{\mathbb{E}} Y_{n i}\right\}$ satisfies the conditions of Lemma
1. Applying Lemma 1 with $x=(\varepsilon / 6), d>0$, and (30), taking $q>p$, we have

$$
\begin{aligned}
& I_{22} \ll \sum_{n=1}^{\infty} n^{p-2} \mathbb{V}\left(\sum_{i=1}^{n} a_{n i}\left(Y_{n i}-\hat{\mathbb{E}} Y_{n i}\right)>\frac{\varepsilon}{6}\right) \\
& +g(n) \exp \left(\frac{\varepsilon}{6 d}-\frac{\varepsilon}{6 d} \ln \left(1+\frac{\varepsilon d}{6 \sum_{i=1}^{n} \hat{\mathbb{E}}\left|a_{n i}\left(Y_{n i}-\hat{\mathbb{E}} Y_{n i}\right)\right|^{2}}\right)\right)=I_{221}+I_{222} . \\
& \left|Y_{n i}\right|^{r} \leq\left|X_{i}\right|^{r} I\left(\left|X_{i}\right| \leq n\right)+n^{r} I\left(\left|X_{i}\right|>n\right) \\
& \leq\left|X_{i}\right|^{r} z\left(\frac{\mu\left|X_{i}\right|}{n}\right)+n^{r}\left(1-z\left(\frac{\left|X_{i}\right|}{n}\right)\right) .
\end{aligned}
$$$$
\leq \sum_{n=1}^{\infty} n^{p-2}\left(\sum_{i=1}^{n} \frac{1}{d^{q}} \hat{\mathbb{E}}\left|a_{n i}\left(Y_{n i}-\hat{\mathbb{E}} Y_{n i}\right)\right|^{q}\right.
$$$$
z_{j}\left(\frac{X}{2^{j}}\right) \leq I\left(\mu 2^{(j-1)}<|X|<(1+\mu) 2^{j}\right),
$$

Firstly, we prove $I_{221}<\infty$. For any $r>0$, by the $\mathrm{Cr}$ in-

Thus,

$$
\begin{aligned}
\hat{\mathbb{E}}\left|Y_{n i}\right|^{r} & \leq \hat{\mathbb{E}}\left(\left|X_{i}\right|^{r} z\left(\frac{\mu\left|X_{i}\right|}{n}\right)\right)+n^{r} \hat{\mathbb{E}}\left(1-z\left(\frac{\left|X_{i}\right|}{n}\right)\right) \\
& =\hat{\mathbb{E}}\left(|X|^{r} z\left(\frac{\mu|X|}{n}\right)\right)+n^{r} \hat{\mathbb{E}}\left(1-z\left(\frac{|X|}{n}\right)\right) \\
& \leq \hat{\mathbb{E}}\left(|X|^{r} z\left(\frac{\mu|X|}{n}\right)\right)+n^{r} \mathbb{V}(|X|>\mu n) .
\end{aligned}
$$

For $I_{221}$, it follows from (42) and (22) that

$$
\begin{aligned}
I_{221} & \leq c \sum_{n=1}^{\infty} n^{p-2} \sum_{i=1}^{n} a_{n i}^{q} \hat{\mathbb{E}}\left|Y_{n i}\right|^{q} \\
& \leq c \sum_{n=1}^{\infty} n^{p-2} \sum_{i=1}^{n} a_{n i}^{q}\left(\hat{\mathbb{E}}\left(|X|^{q} z\left(\frac{\mu|X|}{n}\right)\right)+n^{q} \mathbb{V}(|X|>\mu n)\right) \\
& \leq c \sum_{n=1}^{\infty} n^{p-2}\left(\max _{1 \leq i \leq n} a_{n i}\right)^{q} \sum_{i=1}^{n} 1\left(\hat{\mathbb{E}}\left(|X|^{q} z\left(\frac{\mu|X|}{n}\right)\right)+n^{q} \mathbb{V}(|X|>\mu n)\right) \\
& \leq c \sum_{n=1}^{\infty} n^{p-1-q}\left(\hat{\mathbb{E}}\left(|X|^{q} z\left(\frac{\mu|X|}{n}\right)\right)+n^{q} \mathbb{V}(|X|>\mu n)\right) \\
& \ll \sum_{n=1}^{\infty} n^{p-1-q} \hat{\mathbb{E}}\left(|X|^{q} z\left(\frac{\mu|X|}{n}\right)\right)+\sum_{n=1}^{\infty} n^{p-1} \mathbb{V}(|X|>\mu n) \\
& =: I_{221}^{\prime}+I_{221}^{\prime \prime} .
\end{aligned}
$$

When $p>1$, we can get $I_{221}^{\prime \prime}<\infty$ by (16). Next, we prove $I_{221}^{\prime}<\infty$.

Let $z_{j}(x)$ be an even function and $z_{j}(x) \in C_{l, \text { Lip }}(\mathbb{R})$, $j \geq 1$ such that $0 \leq z_{j}(x) \leq 1$ or all $x$ and $z_{j}\left(x / 2^{j}\right)=1$ if $2^{j-1}<|x| \leq 2^{j} \quad$ and $\quad z_{j}\left(x / 2^{j}\right)=0$ if $|x| \leq \mu 2^{j-1} \quad$ or $|x|>(1+\mu) 2^{j}$. Then,

$$
|X|^{r} z\left(\frac{X}{2^{k}}\right) \leq 1+\sum_{j=1}^{k}|X|^{r} z_{j}\left(\frac{X}{2^{j}}\right), \quad \text { for } \forall r>0
$$

For every $n$, there exists $k$ such that $2^{k-1} \leq n<2^{k}$; thus, by (8) and (44) and from $z(x)$ which is nonincreasing for any $x>0$, we obtain

$$
\begin{aligned}
I_{221}^{\prime} & =\sum_{k=1}^{\infty} \sum_{2^{k-1} \leq n<2^{k}} 2^{k(p-1-q)} \hat{\mathbb{E}}\left(|X|^{q} z\left(\frac{\mu|X|}{2^{k}}\right)\right) \\
& \ll \sum_{k=1}^{\infty} 2^{k(p-q)} \hat{\mathbb{E}}\left(|X|^{q} z\left(\frac{\mu|X|}{2^{k}}\right)\right) \\
& \ll \sum_{k=1}^{\infty} 2^{k(p-q)} \sum_{j=1}^{k} \hat{\mathbb{E}}\left(|X|^{q} z_{j}\left(\frac{\mu|X|}{2^{j}}\right)\right) \\
& \ll \sum_{k=1}^{\infty} 2^{k(p-q)} \sum_{j=1}^{k} 2^{q j} \hat{\mathbb{E}}\left(z_{j}\left(\frac{\mu|X|}{2^{j}}\right)\right) \\
& =\sum_{j=1}^{\infty} 2^{q j} \hat{\mathbb{E}}\left(z_{j}\left(\frac{\mu|X|}{2^{j}}\right)\right) \sum_{k=j}^{\infty} 2^{k(p-q)} \\
& \ll \sum_{j=1}^{\infty} 2^{p j} \hat{\mathbb{E}}\left(z_{j}\left(\frac{\mu|X|}{2^{j}}\right)\right) \\
& \leq \sum_{j=1}^{\infty} 2^{p j} \mathbb{V}\left(|X|>\mu 2^{(j-1)}\right) \\
& =\sum_{j=1}^{\infty}\left(2^{p}\right)^{j} \mathbb{V}\left(|X|>\mu\left(2^{p}\right)^{(j / p)}\right)<\infty .
\end{aligned}
$$

It follows that $I_{221}<\infty$. For $I_{222}$, we consider the following two cases. 
Case 2. $p \geq 2$.

By (8), we have $\hat{\mathbb{E}}\left|Y_{n i}\right|^{2} \leq C_{\mathbb{V}}\left(\left|Y_{n i}\right|^{2}\right) \leq C_{\mathbb{V}}\left(|X|^{2}\right)<\infty$, $\tau<1<1+\gamma$, and (26), and taking $d=(\varepsilon /(12(p+\gamma)))$ and (A.1) or (A.2) hold that

$$
\begin{aligned}
& I_{222} \leq c \sum_{n=1}^{\infty} n^{p-2} g(n) \exp \left(-\frac{\varepsilon}{6 d} \ln \left(\frac{\varepsilon d}{c \sum_{i=1}^{n} a_{n i}^{2}}\right)\right) \\
& \leq c \sum_{n=1}^{\infty} n^{p-2} g(n) \exp \left(-\frac{\varepsilon}{6 d} \ln \left(\frac{\varepsilon d}{c n^{-1}}\right)\right) \\
& \leq c \sum_{n=1}^{\infty} n^{p-2} g(n) n^{-(\varepsilon / 6 d)} \\
& \leq\left\{\begin{array}{l}
c \sum_{n=1}^{\infty} n^{p-2+\tau-(\varepsilon / 6 d)}, \quad \text { if (A.1)holds, } \\
c \sum_{n=1}^{\infty} n^{p-2-(\varepsilon / 6 d)} \frac{g(n) h^{\gamma}(n) n^{\gamma+1}}{n h^{\gamma}(n) n^{\gamma}}, \quad \text { if (A.2)holds, }
\end{array}\right. \\
& \leq \begin{cases}c \sum_{n=1}^{\infty} n^{p-2+\tau-(\varepsilon / 6 d)}, & \text { if (A.1)holds } \\
c \sum_{n=1}^{\infty} n^{p-1-(\varepsilon / 6 d)+\gamma}, & \text { if (A.2)holds }\end{cases} \\
& = \begin{cases}c \sum_{n=1}^{\infty} n^{p-2+\tau-(\varepsilon /(6(\varepsilon /(12(p+\gamma))))}, & \text { if (A.1)holds, } \\
c \sum_{n=1}^{\infty} n^{p-1+\gamma-(\varepsilon /(6(\varepsilon /(12(p+\gamma))))}, & \text { if (A.2)holds }\end{cases} \\
& \ll \sum_{n=1}^{\infty} n^{-1-p-\gamma}<\infty .
\end{aligned}
$$

Case 3. $1<p<2$.

$$
\text { Similar to Case 2, taking }
$$
$d=(\varepsilon /(12(1+((\gamma+1) /(p-1)))))$ and (A.1) or (A.2) hold, we have

$$
\begin{aligned}
& I_{222} \leq c \sum_{n=1}^{\infty} n^{p-2} g(n) \exp \left(-\frac{\varepsilon}{6 d} \ln \left(\frac{\varepsilon d}{6 \sum_{i=1}^{n} a_{n i}^{2} \hat{\mathbb{E}}\left|Y_{n i}\right|^{p}\left|Y_{n i}\right|^{2-p}}\right)\right) \\
& \leq c \sum_{n=1}^{\infty} n^{p-2} g(n) \exp \left(-\frac{\varepsilon}{6 d} \ln \left(\frac{\varepsilon d}{c n^{2-p} \sum_{i=1}^{n} a_{n i}^{2}}\right)\right) \\
& \leq c \sum_{n=1}^{\infty} n^{p-2} g(n) \exp \left(-\frac{\varepsilon}{6 d} \ln \left(\frac{\varepsilon d}{c n^{1-p}}\right)\right) \\
& \leq c \sum_{n=1}^{\infty} n^{p-2} g(n) n^{-(\varepsilon / 6 d)(p-1)} \\
& \leq\left\{\begin{array}{l}
c \sum_{n=1}^{\infty} n^{p-2+\tau-(\varepsilon / 6 d)(p-1)}, \quad \text { if (A.1)holds, } \\
c \sum_{n=1}^{\infty} n^{p-2-(\varepsilon / 6 d)(p-1)} \frac{g(n) h^{\gamma}(n) n^{\gamma+1}}{n h^{\gamma}(n) n^{\gamma}}, \quad \text { if (A.2)holds, }
\end{array}\right. \\
& \leq \begin{cases}c \sum_{n=1}^{\infty} n^{p-2+\tau-(\varepsilon / 6 d)(p-1)}, & \text { if (A.1)holds, } \\
c \sum_{n=1}^{\infty} n^{p-1-(\varepsilon / 6 d)(p-1)+\gamma}, & \text { if (A.2)holds, }\end{cases} \\
& = \begin{cases}c \sum_{n=1}^{\infty} n^{p-2+\tau-(\varepsilon /(6(\varepsilon /(12(1+((\gamma+1) /(p-1))))))(p-1)}, & \text { if (A.1)holds, } \\
c \sum_{n=1}^{\infty} n^{p-1+\gamma-(\varepsilon /(6(\varepsilon /(12(1+((\gamma+1) /(p-1))))))(p-1)}, & \text { if (A.2)holds, }\end{cases} \\
& \ll \sum_{n=1}^{\infty} n^{-1-p-\gamma}<\infty
\end{aligned}
$$

So, when $p>1$, we have $I_{22}<\infty$.

Case 4. $p=1$.

Let $x=(\varepsilon / 6)$ and $d=(\varepsilon \delta /(12(1+\gamma)))$ in Lemma 1; taking $q>p$ and (30), we still can obtain (40). And, similar to Case 1, we also get $I_{221}<\infty$. Next, we discuss the situation of (A.1) or (A.2) holds.

If (A.1) holds, according to (19), we can get $\hat{\mathbb{E}}\left|Y_{n i}\right|^{1+\delta} \leq C_{\mathbb{V}}\left(|X|^{1+\delta}\right)<\infty$. Then, 


$$
\begin{aligned}
I_{22} & \ll \sum_{n=1}^{\infty} n^{-1} g(n) \exp \left(\frac{\varepsilon}{6 d}-\frac{\varepsilon}{6 d} \ln \left(1+\frac{\varepsilon d}{6 \sum_{i=1}^{n} \hat{\mathbb{E}}\left|a_{n i}\left(Y_{n i}-\hat{\mathbb{E}} Y_{n i}\right)\right|^{2}}\right)\right) \\
& \leq c \sum_{n=1}^{\infty} n^{-1} g(n) \exp \left(-\frac{\varepsilon}{6 d} \ln \left(\frac{\varepsilon d}{c \sum_{i=1}^{n} a_{n i}^{2} \hat{\mathbb{E}}\left|Y_{n i}\right|^{2}}\right)\right) \\
& \leq c \sum_{n=1}^{\infty} n^{-1} g(n) \exp \left(-\frac{\varepsilon}{6 d} \ln \left(\frac{\varepsilon d}{c \sum_{i=1}^{n} a_{n i}^{2} \hat{\mathbb{E}}\left|Y_{n i}\right|^{1+\delta}\left|Y_{n i}\right|^{1-\delta}}\right)\right) \\
& \leq c \sum_{n=1}^{\infty} n^{-1} g(n) \exp \left(-\frac{\varepsilon}{6 d} \ln \left(\frac{\varepsilon d}{c n^{1-\delta} \sum_{i=1}^{n} a_{n i}^{2}}\right)\right) \\
& \leq c \sum_{n=1}^{\infty} n^{-1} g(n) \exp \left(-\frac{\varepsilon}{6 d} \ln \left(\frac{\varepsilon d}{c n^{-\delta}}\right)\right) \\
& \leq c \sum_{n=1}^{\infty} n^{-1} g(n) n^{-(\varepsilon / 6 d) \delta} \leq c \sum_{n=1}^{\infty} n^{-1+\tau} n^{-(\varepsilon / 6 d) \delta} \\
& \leq c \sum_{n=1}^{\infty} n^{-1+\tau-(\varepsilon / 6 d) \delta}=c \sum_{i=1}^{\infty} n^{-1+\tau-(\varepsilon /(6(\varepsilon \delta /(12(1+\gamma))))) \delta} \\
& \leq c \sum_{n=1}^{\infty} n^{-2-\gamma}<\infty .
\end{aligned}
$$

If (A.2) holds, by (42) and Cr inequality, we have

$$
\begin{aligned}
I_{22} \leq & c \sum_{n=1}^{\infty} n^{-1} g(n) \exp \left(-\frac{\varepsilon}{6 d} \ln \left(\frac{\varepsilon d}{c \sum_{i=1}^{n} a_{n i}^{2} \hat{\mathbb{E}}\left|Y_{n i}\right|^{2}}\right)\right) \\
\ll & \sum_{n=1}^{\infty} n^{-1} g(n)\left(\sum_{i=1}^{n} a_{n i}^{2} \hat{\mathbb{E}}\left|Y_{n i}\right|^{2}\right)^{(\varepsilon / 6 d)} \\
\leq & \sum_{n=1}^{\infty} n^{-1-(\varepsilon / 6 d)} g(n)\left(\hat{\mathbb{E}}|X|^{2} z\left(\frac{\mu|X|}{n}\right)+n^{2} \hat{\mathbb{E}}\left(1-z\left(\frac{|X|}{n}\right)\right)\right)^{(\varepsilon / 6 d)} \\
\leq & \sum_{n=1}^{\infty} n^{-1-(\varepsilon / 6 d)} g(n)\left(\hat{\mathbb{E}}|X|^{2} z\left(\frac{\mu|X|}{n}\right)\right)^{(\varepsilon / 6 d)} \\
& +\sum_{n=1}^{\infty} n^{-1-(\varepsilon / 6 d)} g(n)\left(n^{2} \hat{\mathbb{E}}\left(1-z\left(\frac{|X|}{n}\right)\right)\right)^{(\varepsilon / 6 d)} \\
= & I_{4}+I_{5} .
\end{aligned}
$$

Then, in order to prove $I_{22}<\infty$, it suffices to show $I_{4}<\infty$ and $I_{5}<\infty$.

By (36), we can obtain $z(\mu|X| / n) \leq I(|X| \leq(n / \mu))$. Since $h(x) \uparrow$ and $h(x) / x \downarrow$, we can get $x h(x) \uparrow$ and $x / h(x) \uparrow$. Combine $\sum_{n=1}^{\infty} g(n) /\left(n h^{\gamma}(\mu n)\right)<\infty$ and (20), and taking $d=(\varepsilon / 6 \gamma)$, we can obtain

$$
\begin{aligned}
I_{4} & =\sum_{n=1}^{\infty} n^{-1-\gamma} g(n)\left(\hat{\mathbb{E}} \frac{|X| h(|X|)|X|}{h(|X|)} z\left(\frac{\mu|X|}{n}\right)\right)^{\gamma} \\
& \leq \sum_{n=1}^{\infty} n^{-1-\gamma} g(n)\left(\frac{(n / \mu)}{h(n / \mu)} \hat{\mathbb{E}}|X| h(|X|)\right)^{\gamma} \\
& \ll \sum_{n=1}^{\infty} \frac{g(n)}{n h^{\gamma}(\mu n)}(\hat{\mathbb{E}}|X| h(|X|))^{\gamma} \\
& <\infty .
\end{aligned}
$$

And, by (36), we also can get $1-z(|X| / n) \leq I(|X| \geq \mu n)$. And, combine $x / h(x) \uparrow$; then,

$$
\begin{aligned}
I_{5} & =\sum_{n=1}^{\infty} n^{-1-\gamma} g(n)\left(n^{2} \hat{\mathbb{E}} \frac{|X| h(|X|)}{|X| h(|X|)}\left(1-z\left(\frac{|X|}{n}\right)\right)\right)^{\gamma} \\
& \leq \sum_{n=1}^{\infty} n^{-1-\gamma} g(n)\left(n^{2} \hat{\mathbb{E}} \frac{|X| h(|X|)}{(\mu n) h(\mu n)}\right)^{\gamma} \\
& \ll \sum_{n=1}^{\infty} n^{-1-\gamma} g(n)\left(n \frac{\hat{\mathbb{E}}|X| h(|X|)}{h(\mu n)}\right)^{\gamma} \\
& =\sum_{n=1}^{\infty} n^{-1} g(n)\left(\frac{\hat{\mathbb{E}}|X| h(|X|)}{h(\mu n)}\right)^{\gamma} \\
& =\sum_{n=1}^{\infty} \frac{g(n)}{n h^{\gamma}(\mu n)}(\hat{\mathbb{E}}|X| h(|X|))^{\gamma} \\
& <\infty .
\end{aligned}
$$

So, we have proved $I_{22}<\infty$.

Next, we prove $I_{3}<\infty$. From (3), (22), and (27), we have

$$
\begin{aligned}
\sum_{i=1}^{n} a_{n i}\left|\hat{\mathbb{E}} X_{i}^{\prime}-\hat{\mathbb{E}} X_{i}\right| & \leq \sum_{n=1}^{n} a_{n i} \hat{\mathbb{E}}\left|X_{i}^{\prime}-X_{i}\right| \\
& \leq \max _{1 \leq i \leq n} a_{n i} \sum_{n=1}^{n} \hat{\mathbb{E}}|X| I(|X|>N) \\
& \leq \hat{\mathbb{E}}|X| I(|X|>N)<\frac{\varepsilon}{6} .
\end{aligned}
$$

So, we can obtain $\mathbb{V}\left(\sum_{i=1}^{n} a_{n i}\left(\hat{\mathbb{E}} X_{i}^{\prime}-\hat{\mathbb{E}} X_{i}\right)>(\varepsilon / 3)\right)=0$, when $n>N$. It follows that $I_{3}=\sum_{n=1}^{\infty} n^{p-2} \mathbb{V}\left(\sum_{i=1}^{n} a_{n i}\right.$ $\left.\left(\hat{\mathbb{E}} X_{i}^{\prime}-\hat{\mathbb{E}} X_{i}\right)>(\varepsilon / 3)\right)<\infty$. Then, (23) holds.

Obviously, $\left\{-X_{n}, n \geq 1\right\}$ also satisfies the conditions of Theorem 1. Considering $\left\{-X_{n}, n \geq 1\right\}$ instead of $\left\{X_{n}, n \geq 1\right\}$ in (23), we can obtain (24).

In particular, if $\widehat{E} X_{i}=\widehat{\varepsilon} X_{i}$, then (25) follows from (23), and 


$$
\begin{aligned}
\mathbb{V}\left(\left|\sum_{i=1}^{n} a_{n i}\left(X_{i}-\hat{\varepsilon} X_{i}\right)\right|>\varepsilon\right) & \\
& \mathbb{V}\left(\sum_{i=1}^{n} a_{n i}\left(X_{i}-\hat{\varepsilon} X_{i}\right)>\varepsilon\right)+\mathbb{V}\left(\sum_{i=1}^{n} a_{n i}\left(X_{i}-\hat{\varepsilon} X_{i}\right)<-\varepsilon\right) .
\end{aligned}
$$

Together with (23)-(25) holds. The proof of Theorem 1 is completed.

We give an example satisfying the conditions of Theorem 1 and obtain corresponding results.

Example 1. Let $\left\{X_{n} ; n \geq 1\right\}$ be a sequence of WA and identically distributed random variables with dominating coefficient $g(n)=n^{(1 / 2)}$ under sublinear expectations and $\mathbb{E}$ is countably subadditive. Assume $a_{n i}=(i n)^{-1}$ and $\mathbb{V}(|X|>x) \sim\left(1 / x^{1+\delta_{1}}\right)$ for some $\delta_{1}>0$. Then, for all $\varepsilon>0$, it will show that

$$
\begin{gathered}
\sum_{n=1}^{\infty} n^{-1} \mathbb{V}\left(\sum_{i=1}^{n}(i n)^{-1}\left(X_{i}-\hat{\mathbb{E}} X_{i}\right)>\varepsilon\right)<\infty, \\
\sum_{n=1}^{\infty} n^{-1} \mathbb{V}\left(\sum_{i=1}^{n}(i n)^{-1}\left(X_{i}-\hat{\varepsilon} X_{i}\right)<-\varepsilon\right)<\infty .
\end{gathered}
$$

If $\hat{\mathbb{E}} X_{i}=\widehat{\varepsilon} X_{i}$, then

$$
\sum_{n=1}^{\infty} n^{-1} \mathbb{V}\left(\left|\sum_{i=1}^{n}(i n)^{-1}\left(X_{i}-\hat{\mathbb{E}} X_{i}\right)\right|>\varepsilon\right)<\infty .
$$

Obviously, $g(x)=x^{(1 / 2)}$ satisfies (A.1). $\exists 0<\delta<\delta_{1}$, we have $C_{\mathbb{V}}\left(|X|^{1+\delta}\right) \sim \int_{1}^{\infty} \mathbb{V} \quad\left(|X|_{\hat{\mathbb{E}}}^{1+\delta}>x\right) \mathrm{d} x \sim \int_{1}^{\infty}\left(1 / x^{\left(\left(1+\delta_{1}\right) /\right.}\right.$ $(1+\delta))) \mathrm{d} x<\infty$. Furthermore, $\hat{\mathbb{E}}$ is countably subadditive, and by [6], then we have $\hat{\mathbb{E}}\left(|X|^{1+\delta}\right) \leq C_{\mathbb{V}}\left(|X|^{1+\delta}\right)<\infty$. Therefore, all the conditions of Theorem 1 are satisfied; then, Theorem 1 holds.

\section{Data Availability}

No data were used to support this study.

\section{Conflicts of Interest}

The authors declare that they have no conflicts of interest.

\section{Acknowledgments}

This work was supported by the National Natural Science Foundation of China (12061028) and Support Program of the Guangxi China Science Foundation (2018GXNSFAA281011).

\section{References}

[1] S. G. Peng, "G-Brownian motion and related stochastic calculus of Ito type," Stochastic Analysis and Applications, vol. 34, no. 2, pp. 139-161, 2007.
[2] S. Peng, "Multi-dimensional G-Brownian motion and related stochastic calculus under G-expectation," Stochastic Processes and Their Applications, vol. 118, no. 12, pp. 2223-2253, 2008.

[3] S. G. Peng, "A new central limit theorem under sublinear expectations," Journal of Mathematics, vol. 53, no. 8, pp. 1989-1994, 2008.

[4] L. X. Zhang, "Strong limit theorems for extended independent and extended negatively dependent random variables under non-linear expectations," 2016, https://arxiv.org/abs/1608. 00710.

[5] L. Zhang, "Exponential inequalities under the sub-linear expectations with applications to laws of the iterated logarithm," Science China Mathematics, vol. 59, no. 12, pp. 2503-2526, 2016.

[6] L. Zhang, "Rosenthal's inequalities for independent and negatively dependent random variables under sub-linear expectations with applications," Science China Mathematics, vol. 59, no. 4, pp. 751-768, 2016.

[7] D. Zhang and Z. Chen, "A weighted central limit theorem under sublinear expectations," Communications in Statistics Theory and Methods, vol. 43, no. 3, pp. 566-577, 2014.

[8] F. X. Feng, D. C. Wang, and Q. Y. Wu, "Complete convergence for weighted sums of negatively dependent random variables under the sub-linear expectations," Journal of Statistics - Theory and Methods, vol. 48, pp. 0361-0926, 2018.

[9] R. X. Wang and Q. Y. Wu, "Some types of convergence for negatively dependent random variables under sublinear expectations," Discrete Dynamics in Nature and Society, vol. 2019, Article ID 9037258, 7 pages, 2019.

[10] Z. J. Chen, "Strong laws of large numbers for sub-linear expectations," Journal of Science China Mathematics, vol. 46, no. 15, pp. 7529-7545, 2016.

[11] Z. . w. Liang and Q. Y. Wu, "Theorems of complete convergence and complete integral convergence for END random variables under sub-linear expectations," Journal of Inequalities and Applications, vol. 114, no. 1, 2019.

[12] P. L. Hsu and H. Robbins, "Complete convergence and the law of large numbers," Proceedings of the National Academy of Sciences, vol. 33, no. 2, pp. 25-31, 1947.

[13] H. Y. Liang and C. Su, "Complete convergence for weighted sums of NA sequences," Statistics Probability Letters, vol. 45, no. 1, pp. 85-95, 2008.

[14] S. H. Sung, "Complete convergence for weighted sums of random variables," Statistics Probability Letters, vol. 3, pp. 303-331, 2011.

[15] Q. Y. Wu, "Complete convergence for weighted sums of sequences of negatively dependent random variables," Journal of Probability and Statistics, vol. 2011, Article ID 202015, 16 pages, 2011.

[16] Q. Y. Wu, "A complete convergence theorem for weighted sums of arrays of rowwise negatively dependent random variables," Journal of Inequalities Applications, vol. 50, no. 1, pp. 1-10, 2012.

[17] J. Lita da Silva, "Strong laws of large numbers for arrays of row-wise extended negatively dependent random variables 
with applications," Journal of Nonparametric Statistics, vol. 32, no. 1, pp. 20-41, 2020.

[18] J. J. Lang, T. Y. He, L. Cheng, C. Lu, and X. J. Wang, "Complete convergence for weighted sums of widely orthantdependent random variables and its statistical application," Revista Matemctica Complutense, vol. 45, pp. 1-29, 2020.

[19] A. Kuczmaszewska, "Complete convergence for widely acceptable random variables under the sublinear expectations $\mathrm{C}$ science direct," Journal of Mathematical Analysis and Applications, vol. 484, no. 1, 2009.

[20] X. C. Ma and Q. Y. Wu, "On some conditions for strong law of large numbers for weighted sums of END random variables under sublinear expectations," Discrete Dynamics in Nature and Society, vol. 2019, Article ID 7945431, 8 pages, 2019. 